\title{
Características y evolución de los pacientes con amiloidosis sistémica y compromiso cardíaco
}

\author{
Features and evolution of patients with systemic amyloidosis and cardiac involvement
}

\begin{abstract}
Marcelina Carretero ${ }^{1 *}, M^{a}$ A. Aguirre ${ }^{2}$, Eugenia Villanueva ${ }^{3}$, Elsa Nucifora ${ }^{2,4}$ y $M^{\text {a }}$ Lourdes Posadas-Martínez ${ }^{1,5}$
${ }^{1}$ Servicio de Clínica Médica, Área de Investigación en Medicina Interna; ${ }^{2}$ Servicio de Clínica Médica; ${ }^{3}$ Servicio de Cardiología; ${ }^{4}$ Sección de Hematología; ${ }^{5}$ Departamento de Investigación, Instituto de Medicina Traslacional e Ingeniería Biomédica, CONICET-Instituto Universitario del Hospital Italiano. Hospital Italiano de Buenos Aires, BA, Argentina
\end{abstract}

\begin{abstract}
Resumen
Objetivos: Determinar la prevalencia de amiloidosis cardíaca en pacientes con amiloidosis sistémica. Comparar la supervivencia según sea que presenten o no compromiso cardíaco. Métodos: Cohorte retrospectiva de pacientes con amiloidosis sistémica del Registro Institucional de Amiloidosis del Hospital Italiano de Buenos Aires, entre 2010 y 2019. Se consideró como compromiso cardíaco la presencia de síntomas o imágenes consistentes con amiloidosis no explicado por otras causas. Se evaluó la muerte por todas las causas. Se calculó la sobrevida mediante Kaplan-Meier. Los factores relacionados con mortalidad se evaluaron con un modelo de regresión de Cox. Se evaluó el trasplante cardíaco en un modelo de regresión de riesgo competitivo. Resultados: La prevalencia de compromiso cardíaco fue del 63\%. La incidencia de muerte fue de 14/1,000 personas-meses para el grupo con compromiso cardíaco y de 5/1,000 personas-meses para los pacientes sin compromiso. Los pacientes con compromiso cardíaco tuvieron una sobrevida global a los cinco años de $44 \%$ contra $67 \%$ en los que no tenían compromiso $(p=0.02)$. El HR crudo para el compromiso cardíaco fue de $2.09(p=0.02)$. La edad mostró un HR ajustado de 1.06 ( $p$ <0.01). El modelo de regresión de riesgos competitivos estableció un sub-HR de 1.86 (IC95\%, 0.99-3.49; $p=0.05)$. Conclusiones: El compromiso cardíaco es un factor pronóstico importante en pacientes con amiloidosis.
\end{abstract}

Palabras claves: Amiloidosis. Amiloidosis de cadenas ligeras de las inmunoglobulinas. Amiloidosis familiar. Miocardiopatía restrictiva.

\begin{abstract}
Objective: To estimate the prevalence of cardiac amyloidosis in patients with systemic amyloidosis. Compare survival rates based on whether they show cardiac involvement. Methods: A retrospective cohort study of patients with systemic amyloidosis from the Institutional Amyloidosis Registry of the Hospital Italian of Buenos Aires from 2010 to 2019. Heart involvement is considered to be the presence of symptoms and/or images consistent with amyloidosis, and there is no other reason to explain it. All deaths due to causes were evaluated. The survival rate was estimated by Kaplan-Meier. Cox regression model was used to evaluate factors related to mortality. Heart transplantation was evaluated in a competitive risk regression model. Results: The prevalence of heart involvement is $63 \%$. For the group with heart damage, the death rate was 14/1,000 person-months, and for patients without damage, the death rate was 5/1,000 person-months. The 5-year overall survival rate for patients with heart involvement was $44 \%$, while that for patients without damage was $67 \%(p=0.02)$. The original HR for
\end{abstract}

Correspondencia:

*Marcelina Carretero

E-mail: marcelina.carretero@ @ospitalitaliano.org.ar
Disponible en internet: 28-06-2021

Arch Cardiol Mex. 2022;92(1):60-67

www.archivoscardiologia.com

1405-9940 / @ 2021 Instituto Nacional de Cardiología Ignacio Chávez. Publicado por Permanyer. Este es un artículo open access bajo la licencia CC BY-NC-ND (http://creativecommons.org/licenses/by-nc-nd/4.0/). 
heart involvement was $2.09(p=0.02)$. Age showed that HRa was $1.06(p<0.01)$. The sub-HR estimated by the competitive risk regression model are $1.86(95 \% \mathrm{Cl} 0.99-3.49) p=0.05$. Conclusion: Cardiac involvement is an important prognostic factor in patients with amyloidosis.

Keywords: Amyloidosis. Immunoglobulin light chain amyloidosis. Familial amyloidosis. Restrictive cardiomyopathy.

\section{Introducción}

La afectación cardíaca es la principal causa de morbimortalidad en la amiloidosis y es frecuente en la amiloidosis sistémica de cadenas ligeras $(A L)$ y la amiloidosis por depósito de transtiretina, sea ésta de tipo familiar o senil (ATTRv, ATTRwt) ${ }^{1,2}$.

La amiloidosis representa un grupo de enfermedades producidas por el mal plegamiento de una proteína, que se acumula en el espacio extracelular en forma de fibrillas insolubles en diversos tejidos y órganos ${ }^{3,4}$. Existen más de 30 proteínas que pueden formar fibrillas amiloides in vivo ${ }^{1,5,6}$. Las manifestaciones clínicas dependen del sitio de los depósitos de las fibrillas amiloides y la proteína precursora que interviene en la formación de las fibrillas ${ }^{7}$.

La incidencia de amiloidosis es variable e incierta. Las formas sistémicas más frecuentes son AL, ATTRv y ATTRwt y la amiloidosis secundaria (AA). La AL es la más frecuente en los países desarrollados, con una incidencia anual de 6 a 12 casos por millón de habitantes en el Reino Unido y EE.UU.1,8-10 La ATTRv posee gran diversidad geográfica. La mutación Val50Met tiene una prevalencia global $<1$ por cada 100,000 habitantes pero es endémica en países como Portugal, Japón y Suecia. La mutación Val142lle está presente en un 4\% de la población de raza negra de Norteamérica ${ }^{11}$. La incidencia de ATTRwt es difícil de precisar y se identifican depósitos de amiloide cardíaco en el $25 \%$ de las necropsias de los pacientes mayores de 80 años $^{12}$. La AA es una entidad poco frecuente. En EE.UU. sólo el $7 \%$ de las biopsias renales con diagnóstico de amiloidosis pertenece a $A A^{13}$. En los países en vías de desarrollo, donde se registra una mayor prevalencia de enfermedades infecciosas insuficientemente tratadas, es probable que la incidencia de la amiloidosis AA sea elevada. Sin embargo, existen escasos datos en Argentina y América Latina acerca de la incidencia de la amiloidosis. El grupo de trabajo de amiloidosis del Hospital Italiano de Buenos Aires realizó el primer estudio epidemiológico en Latinoamérica para determinar la densidad de incidencia de amiloidosis ATTRwt, AL y AA. La densidad de incidencia se calculó en 14/1,000,000 pacientes/año en ATTRwt, 11/1,000,000 pacientes/año en $A L$ y 2/1,000,000 pacientes/año en $A A^{14}$.
La frecuencia de compromiso cardíaco varía según sea el tipo de amiloidosis. En la $A L$, la afectación cardíaca se identifica hasta en el $60 \%$ de los pacientes ${ }^{7,15,16}$. En la ATTRv, la afectación cardíaca varía de acuerdo con la mutación que causa la enfermedad: es poco frecuente en la mutación V50M y del $90 \%$ en los pacientes con la mutación T60A ${ }^{17}$. La ATTRwt, o amiloidosis sistémica senil, predomina en los hombres, por lo general mayores de 70 años, y afecta al corazón en el $100 \%$ de los casos ${ }^{1,16,18,19}$. La AA lesiona en especial al riñón, con casi $2 \%$ de compromiso cardíaco ${ }^{1,7}$.

La afectación cardíaca es un factor pronóstico en la amiloidosis sistémica. La mediana de supervivencia de los pacientes con $\mathrm{AL}$ y la afectación cardíaca puede llegar hasta los tres años ${ }^{9}$, pero cuando el compromiso cardíaco es grave, la mediana de supervivencia es de seis a 12 meses ${ }^{10,16,17}$. Los pacientes con ATTRv y ATTRwt tienen mejor pronóstico que aquéllos con Al y es posible llegar hasta los seis años de sobrevida ${ }^{17,20}$. Sin embargo, cuando los depósitos de amiloide cardíacos son extensos, pueden conducir a una insuficiencia cardíaca de relevancia clínica ${ }^{16}$.

En Latinoamérica en general, y en Argentina en particular, el conocimiento sobre la amiloidosis cardíaca y su prevalencia y pronóstico es limitado. En el Hospital Italiano de Buenos Aires se cuenta con un Registro Institucional de Amiloidosis que incluye activamente a pacientes con amiloidosis de cualquier tipo desde el año 2010. El objetivo es determinar la prevalencia de amiloidosis cardíaca en pacientes con amiloidosis sistémica incluidos en el Registro Institucional de Amiloidosis, comparar la supervivencia según sea que presenten o no compromiso cardíaco, y valorar los factores relacionados con mortalidad por todas las causas.

\section{Materiales y métodos}

\section{Diseño}

Cohorte retrospectiva. Se incluyó de forma consecutiva a todos los pacientes con diagnóstico de amiloidosis sistémica del Registro Institucional de Amiloidosis (RIA) en el Hospital Italiano de Buenos Aires (01/2010-05/2019). 


\section{Ámbito}

El Hospital Italiano de Buenos Aires es una institución universitaria de tercer nivel de complejidad, que recibe a pacientes de todo el territorio argentino y países limítrofes.

El Registro Institucional de Amiloidosis tiene inclusión activa y prospectiva desde el 2010 de todos los casos incidentes de amiloidosis. Funciona de modo transdisciplinario, con la participación de las áreas de Investigación en Medicina Interna, Anatomía Patológica, Laboratorio, Cardiología, Clínica Médica, Hematología, Hepatología, Nefrología y Neurología. Está registrado en Clinical Trials (NCT01347047) y tienen aprobación del comité de ética de protocolos de investigación del hospital con el número de protocolo 1675. Todos los pacientes realizan el proceso de consentimiento informado antes de incluirse en el registro.

\section{Participantes}

Criterios de elegibilidad. Se identificó a todos los pacientes con diagnóstico de amiloidosis incluidos en el Registro Institucional de Amiloidosis del Hospital Italiano de Buenos Aires.

Selección de los pacientes. Se incluyó a los pacientes mayores de 17 años, con diagnóstico de amiloidosis sistémica. Se excluyó a los pacientes con amiloidosis localizada o no tipificada.

\section{Fuente de datos}

La información clínica-demográfica, el tipo de amiloidosis, el compromiso orgánico y los estudios complementarios al diagnóstico se recogieron de manera estandarizada con un formulario de informe de caso como parte del Registro Institucional de Amiloidosis. Se efectuó una revisión de historias clínicas para los datos faltantes o inconsistentes.

\section{Variables/definiciones diagnósticas}

El diagnóstico de la amiloidosis sistémica se definió mediante la documentación histológica con la tinción rojo Congo ${ }^{21}$ en grasa abdominal, glándulas salivares accesorias $u$ órgano afectado o mediante el cuadro clínico consistente y plasmocitos clonales en la médula ósea o bioquímica con cadenas ligeras libres y la relación alterada entre éstas.

La amiloidosis cardíaca se definió con biopsia endomiocárdica positiva para amiloidosis, ante evidencia clínica o bioquímica, o ecocardiograma indicativo de amiloidosis, en presencia de biopsia extracardíaca positiva para amiloidosis ${ }^{21}$ o diagnóstico imagenológico con resonancia cardíaca con contraste intravenoso o centellograma de pirofosfato con hallazgos consistentes con amiloidosis cardíaca.

El compromiso orgánico, renal, nervioso, gastrointestinal, hepático y de partes blandas se definió de acuerdo con el Consenso de Opinión del $10^{\mathrm{a}}$ Simposio Internacional sobre amiloide y amiloidosis ${ }^{21}$.

\section{Seguimiento}

Todos los pacientes recibieron seguimiento de manera estandarizada para evaluar la mortalidad por todas las causas. El seguimiento se realizó desde la fecha del diagnóstico de amiloidosis sistémica hasta el día de la muerte o fecha de trasplante cardíaco o la fecha del último contacto con el sistema de salud o cierre administrativo del estudio (31/05/2019).

\section{Análisis estadístico}

Las variables cuantitativas se describieron con su media y desviación estándar o mediana e intervalo intercuartílico según la distribución. Las variables categóricas se notificaron como frecuencias y porcentajes.

La prevalencia de compromiso cardíaco se determinó como porcentaje y se calcularon los intervalos de confianza del 95\% (IC95\%). La mortalidad por todas las causas se estableció mediante Kaplan-Meier. Se informó la sobrevida para cada tiempo con su IC95\%. Se comparó la sobrevida de acuerdo con el compromiso cardíaco con la prueba long-rank.

Los factores relacionados con mortalidad se evaluaron con un modelo regresión de Cox, se presentaron los cocientes de riesgo (HR, hazard ratio) crudos y ajustados con sus IC95\%. Las variables de ajuste se definieron a priori según su relevancia clínica y el conocimiento del tema.

De manera adicional se sometió a prueba la relación de tiempo a la muerte a través de un modelo de regresión de riesgos competitivos como una alternativa útil a la regresión de Cox en presencia de trasplante cardíaco como riesgo competitivo. Se calculó el coeficiente relacionado con cada predictor y se presentó con el sub-HR de riesgo y su respectivo IC95\%. Se consideró estadísticamente significativo un valor de $p<0.05$. Se realizó el análisis con el software Stata 14. 
Tabla 1. Características iniciales de los pacientes con amiloidosis sistémica incluidos en el RIA en el período 2010-2019, globales y por tipo de amiloidosis $(n=167)$

\begin{tabular}{|l|c|c|c|c|c|}
\hline Característica & $\begin{array}{c}\text { Global } \\
(\mathbf{n}=\mathbf{1 6 7 )}\end{array}$ & $\begin{array}{c}\text { AL } \\
(\mathbf{n = 8 7})\end{array}$ & $\begin{array}{c}\text { ATTRv } \\
(\mathbf{n}=\mathbf{1 8})\end{array}$ & $\begin{array}{c}\text { ATTRwt } \\
(\mathbf{n}=\mathbf{4 0})\end{array}$ & $\begin{array}{c}\text { AA } \\
(\mathbf{n}=\mathbf{2 2})\end{array}$ \\
\hline Edad*, años (IIC) & $68(54-76)$ & $64(53-72)$ & $61(39-66)$ & $81(74-86)$ & $56(50-64)$ \\
\hline Hombres, $n(\%)$ & $106(64)$ & $46(53)$ & $13(72)$ & $37(93)$ & $10(45)$ \\
\hline Calificación de Charlson* (IIC) & $2(1-3)$ & $2(0-3)$ & $1(1-4)$ & $1(1-3)$ & $2(2-3)$ \\
\hline
\end{tabular}

IIC: intervalo intercuartílico; AL: amiloidosis AL; ATTRv: amiloidosis TTR mutada; ATTRwt: amiloidosis TTR tipo natural; AA: amiloidosis AA. *Mediana.

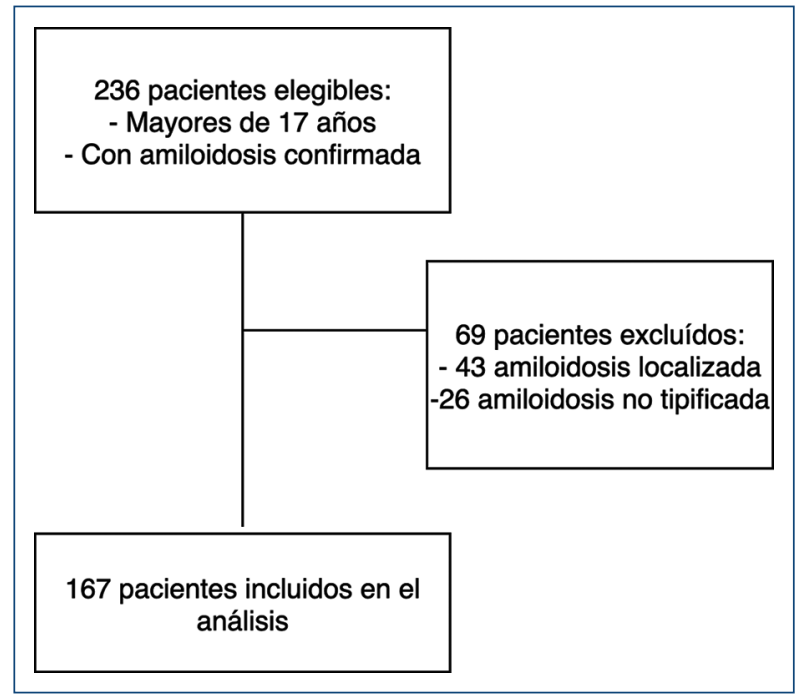

Figura 1. Diagrama de flujo de pacientes con amiloidosis incluidos en el RIA en el período 2010-2019.

\section{Resultados}

\section{Participantes}

En el período de interés, 236 pacientes fueron potencialmente elegibles, excluidos 69 por amiloidosis localizada o amiloidosis no tipificada. Se analizó a 167 pacientes (Fig. 1) de los cuales 87 (52\%) se diagnosticaron con AL, 40 (24\%) con ATTRwt, 22 (13\%) con AA y $18(11 \%)$ con ATTRv.

\section{Datos descriptivos}

Las características iniciales de los pacientes analizados se muestran en la Tabla 1. Hubo un predominio global de hombres y en los diferentes tipos de amiloidosis, excepto para la AA. La mediana de edad fue de 68 años (IIC, 54-76), con amplia variabilidad entre los subtipos de amiloidosis. Los pacientes más añosos pertenecían al grupo de ATTRwt.
Tabla 2. Frecuencia de órganos afectados de pacientes con amiloidosis incluidos en el RIA en el período 2010-2019 $(\mathrm{n}=167)$

\begin{tabular}{|l|c|}
\hline Amiloidosis sistémica $=167$ & $105(63 \%)$ \\
Corazón & $83(50 \%)$ \\
Riñón & $49(29 \%)$ \\
Gastrointestinal & $41(25 \%)$ \\
\hline Nervioso &
\end{tabular}

Las formas más frecuentes de presentación clínica fueron la disnea en 86 (52\%) pacientes, la insuficiencia renal en $73(47 \%)$ y la insuficiencia cardíaca en $68(41 \%)$. La disnea fue la forma más frecuente de presentación en los pacientes con $\mathrm{AL}$, identificada en 50 (57\%). En ATTRwt, 31 (77\%) pacientes iniciaron con disnea y $28(70 \%)$ con insuficiencia cardíaca. En $11(62 \%)$ pacientes con ATTRv, la polineuropatía fue la forma más frecuente de presentación, mientras que en la AA lo fue la insuficiencia renal en 17 (77\%). La frecuencia de órganos afectados en la amiloidosis sistémica se muestra en las Tablas 2 y 3.

Entre los estudios diagnósticos realizados para amiloidosis cardíaca, el ecocardiograma y el electrocardiograma fueron los más empleados. La Tabla 4 muestra los datos de los estudios diagnósticos efectuados para amiloidosis cardíaca.

\section{Resultados principales}

La prevalencia de compromiso cardíaco fue del $63 \%$ ( $n=105 ;$ IC95\%, 55-70). La tasa de incidencia de muerte global fue de 10 por 1,000 personas-meses, con un tiempo en riesgo de 5,302 meses.

La incidencia de muerte fue de 14 por 1,000 personas-meses para el grupo con compromiso cardíaco contra 5 por 1,000 personas-meses para los pacientes sin compromiso cardíaco. En los sujetos con AL, la incidencia de muerte fue de 10 por 1,000 personas-meses, en AA la incidencia de muerte fue de 4 por 1,000 
Tabla 3. Frecuencia de órganos afectados de acuerdo con el tipo de amiloidosis sistémica incluidos en el RIA en el período 2010-2019 ( $n=167)$

\begin{tabular}{|l|c|c|c|c|}
\hline Órgano afectado & AL & ATTRv & ATTRwt & AA \\
\hline Corazón & $55(63 \%)$ & $6(33 \%)$ & $40(100 \%)$ & $4(18 \%)$ \\
\hline Riñón & $55(64 \%)$ & $0(0 \%)$ & $11(28 \%)$ & $17(77 \%)$ \\
\hline Nervioso & $25(29 \%)$ & $11(61 \%)$ & $3(8 \%)$ & $2(9 \%)$ \\
\hline Gastrointestinal & $29(33 \%)$ & $9(50 \%)$ & $5(12 \%)$ & $6(27 \%)$ \\
\hline
\end{tabular}

Tabla 4. Estudios diagnósticos realizados en pacientes con amiloidosis sistémica incluidos en el RIA en el período 2010-2019 ( $\mathrm{n}=167$ )

\begin{tabular}{|l|c|c|}
\hline Estudio realizado & $\begin{array}{c}\text { Totales } \\
\text { realizados }\end{array}$ & $\begin{array}{c}\text { Hallazgos consistentes } \\
\text { con amiloidosis }\end{array}$ \\
\hline Ecocardiograma & 145 & $99(68 \%)$ \\
\hline Electrocardiograma & 126 & $51(60 \%)$ \\
\hline RMC & 114 & $78(68 \%)$ \\
\hline $\begin{array}{l}\text { Centellograma con } \\
\text { pirofosfato }\end{array}$ & 25 & $16(64 \%)$ \\
\hline
\end{tabular}

personas-meses, en ATTRv fue de 2 por 1,000 personas-meses, y en los ATTRwt fue de 19 por 1,000 personas-meses. La Tabla 5 muestra la prevalencia de compromiso cardíaco y tasa de mortalidad por tipo de amiloidosis.

La mediana de sobrevida global, medida desde el momento del diagnóstico confirmado de amiloidosis, fue de 64 meses, y para el grupo con compromiso cardíaco fue de 56 meses. La sobrevida global no ajustada se muestra en la figura 2. Los pacientes con compromiso cardíaco presentaron una sobrevida global al año de 78\% (IC95\% 68-85) y a los 5 años de $44 \%$ (IC95\%, 31-56), mientras que los individuos sin compromiso cardíaco mostraron una sobrevida global al año de $82 \%$ (IC95\%, 70-90) y a los 5 años de $67 \%$ (IC95\%, 48-81) con un valor de $p=0.02$. No se realizó la comparación entre grupos de amiloidosis dada la escasa mortalidad en estos grupos y porque en la aTTRwt el compromiso cardíaco es del $100 \%$.

\section{Factores relacionados con la mortalidad}

El HR crudo para mortalidad en el grupo con compromiso cardíaco fue de 2.09 (IC95\%, 1.12-3.91; $p=0.02$ ). Sin embargo, cuando se ajustó por subtipo de

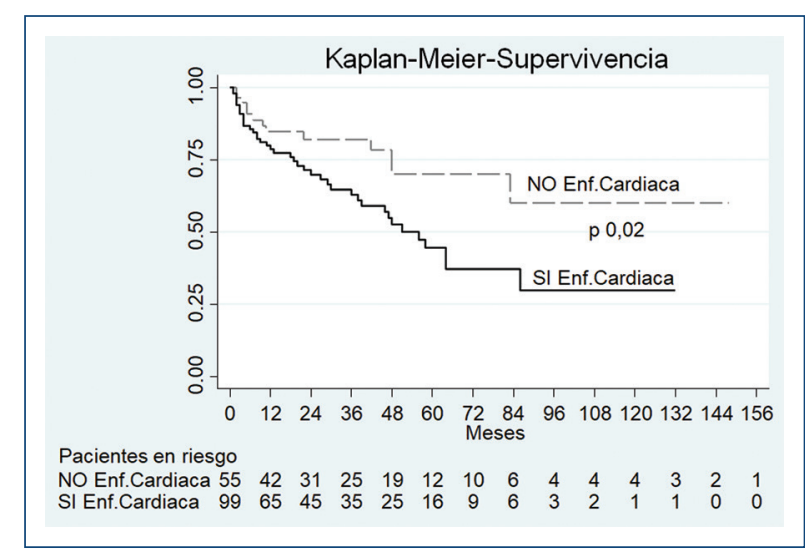

Figura 2. Kaplan-Meier. Curva de sobrevida global de acuerdo con los grupos de compromiso cardíaco de pacientes con amiloidosis incluidos en el RIA en el período 2010-2019, globales y por tipo de amiloidosis. La línea continua representa al grupo con enfermedad cardíaca; la línea punteada representa al grupo sin enfermedad cardiaca.

amiloidosis, edad y compromiso renal, el HR ajustado fue de 1.17 (IC95\%, 0.55-2.5; $p=0.68$ ). La edad tuvo un HR crudo de 1.02 (IC95\%, 1-1.04) con un HR ajustado de 1.06 (IC95\% 1.03-1.09; $p$ <0.01). En la Tabla 6 se muestran los HR crudos y ajustados para cada una de las variables utilizadas en el modelo de regresión.

\section{Riesgos competitivos}

Al evaluar el riesgo de muerte y considerar la presencia de trasplante como evento competitivo entre los grupos con y sin compromiso cardíaco, el sub-HR arrojó un valor de 1.86 (IC95\%, 0.99-3.49; $p=0.05)$. La Figura 3 muestra las curvas de muerte para ambos grupos con trasplante cardíaco como evento competitivo.

\section{Análisis de subgrupo para AL y ATTRwt}

Se realizó un análisis de subgrupo con los subtipos AL y aTTRwt para determinar su relación con la muerte en presencia de trasplante cardíaco como evento competitivo. La Figura 4 muestra las curvas de muerte de ambos subtipos de amiloidosis. Los resultados no mostraron diferencias, con un sub-HR de 1.13, (IC95\%, 0.97-1.32; $p=0.10$ ).

\section{Discusión}

En este estudio se calculó la prevalencia de compromiso cardíaco en pacientes con amiloidosis sistémica y la sobrevida de acuerdo con la presencia o ausencia de compromiso cardíaco. 
Tabla 5. Prevalencia de compromiso cardíaco y tasa de mortalidad de pacientes con amiloidosis incluidos en el RIA en el período 2010-2019 ( $n=167)$

\begin{tabular}{|l|c|c|c|c|c|}
\hline $\begin{array}{l}\text { Tipo de } \\
\text { amiloidosis }\end{array}$ & Total $(\mathbf{n})$ & $\begin{array}{c}\text { Compromiso } \\
\text { cardíaco (n) }\end{array}$ & $\begin{array}{c}\text { Prevalencia de compromiso } \\
\text { cardíaco (IC95\%) }\end{array}$ & Muerte & $\begin{array}{c}\text { Tasa de mortalidad } \\
\text { (IC95\%) }\end{array}$ \\
\hline AL & 87 & 55 & $63 \%(52-73)$ & 33 & $38 \%(28-49)$ \\
\hline ATTRv & 18 & 6 & $33 \%(13-59)$ & 1 & $5 \%(0.1-27)$ \\
\hline ATTRwt & 40 & 40 & $100 \%$ & 21 & $53 \%(36-68)$ \\
\hline AA & 22 & 4 & $18 \%(5-40)$ & 3 & $14 \%(3-35)$ \\
\hline
\end{tabular}

Tabla 6. HR crudos y ajustados para mortalidad de pacientes con amiloidosis incluidos en el RIA en el período 2010-2019 ( $\mathrm{n}=151)$

\begin{tabular}{|l|c|c|c|c|c|c|}
\hline Covariables & HR crudos & IC & p & HR ajustado & IC & p \\
\hline Edad & 1.05 & $1.03-1.07$ & $<0.001$ & 1.06 & $1.03-1.09$ & $<0.001$ \\
\hline Compromiso cardíaco & 2.09 & $1.12-3.91$ & 0.021 & 1.17 & $0.55-2.50$ & 0.688 \\
\hline Compromiso renal & 1.15 & $0.67-1.97$ & 0.611 & 1.28 & $0.71-2.30$ & 0.409 \\
\hline Tipo de amiloidosis comparado con AL & 1 & & & 1 & \\
\hline AA & 0.38 & $0.17-1.26$ & 0.113 & 0.69 & $0.19-2.60$ & 0.587 \\
\hline ATTRut & 1.65 & $0.93-2.95$ & 0.087 & 0.61 & $0.27-1.37$ & 0.230 \\
\hline ATTRm & 0.22 & $0.03-1.61$ & 0.137 & 0.58 & $0.74-4.64$ & 0.611 \\
\hline
\end{tabular}

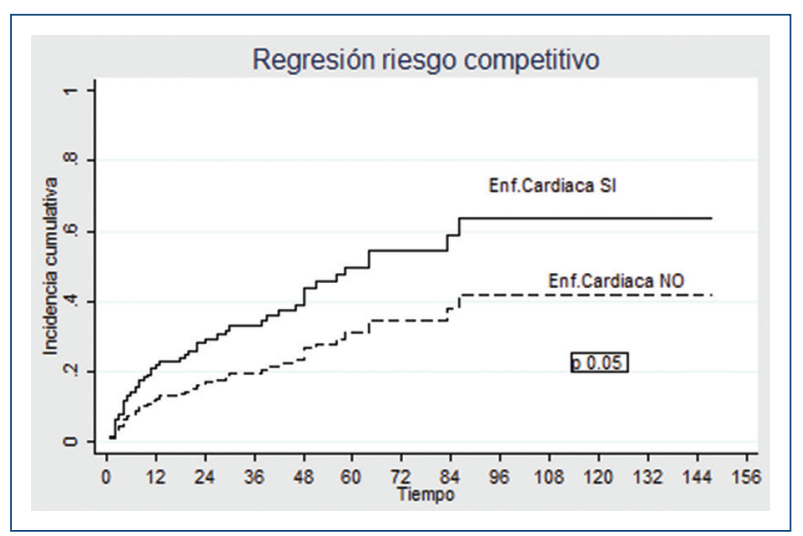

Figura 3. Curva de incidencia cumulativa en presencia de trasplante cardíaco como evento competitivo de acuerdo con grupos de compromiso cardíaco sí/no (RIA 2010-2019).

Los datos arrojaron que los pacientes con amiloidosis sistémica que sufrían compromiso cardíaco tuvieron peor pronóstico que aquéllos sin compromiso cardíaco. La diferencia en la sobrevida global es mayor durante el seguimiento a cinco años. Esto podría explicarse en parte porque, a medida que el paciente

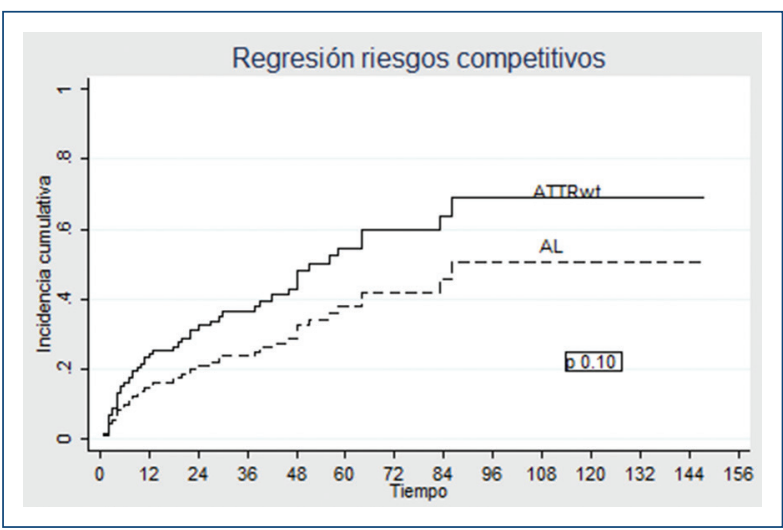

Figura 4. Curva de incidencia cumulativa en presencia de trasplante cardíaco como evento competitivo según tipos de amiloidosis (RIA 2010-2019).

envejece, los factores de riesgo para la enfermedad cardiovascular aumentan y actúan de manera sinérgica con la afectación cardíaca que produce la amiloidosis.

La prevalencia de amiloidosis cardíaca en esta cohorte fue más elevada que la de otros informes. Lee, 
et al. registraron una frecuencia de amiloidosis cardíaca del $48 \%$, en su mayoría pacientes con el subtipo $A L^{22}$. Rapezzi, et al., en su estudio comparativo entre los subtipos de amiloidosis, publicaron una frecuencia de amiloidosis cardíaca del $47 \%{ }^{17}$. Estos estudios son de los años 2013 y 2009, respectivamente. En un protocolo realizado por el grupo de los autores del año 2014, la prevalencia de compromiso cardíaco en pacientes con amiloidosis sistémica fue del $47 \%{ }^{23}$.

La sobrevida global fue menor en los pacientes con amiloidosis sistémica y compromiso cardíaco respecto de los no afectados. Esto es similar con los resultados de Czobor, et al. ${ }^{24}$ que notificaron una tasa de sobrevida de los pacientes con amiloidosis sistémica y afectación cardíaca de 35\% a los cinco años y los de Arnt, et al. que informaron una tasa de sobrevida a los tres años del $63 \%{ }^{25}$ para AL y $83 \%$ para aTTRv. En este estudio, el porcentaje de amiloidosis AL fue de $73 \%$ y no se estudiaron los subtipos aTTRwt y AA.

En cuanto a los factores relacionados con mortalidad, el compromiso cardíaco y la edad mostraron significación estadística en el análisis univariado, mientras que sólo la edad permaneció significativa en el análisis multivariado.

El análisis de regresión de riesgos competitivos que evaluó la muerte global con trasplante cardíaco como evento competitivo mostró diferencias según fuera el compromiso cardíaco.

Este trabajo muestra potenciales limitaciones. La falta de tamaño muestral pudo afectar el hallazgo de diferencias en el análisis multivariado para compromiso cardíaco y evaluar la mortalidad diferente por subtipos de amiloidosis. Las variables adicionales que podrían modificar el pronóstico de la enfermedad, como el tipo de tratamiento recibido y la valoración de biomarcadores cardíacos como el proBNP y la troponina I, no se evaluaron en este estudio. Los datos presentados aquí pueden servir para trabajos futuros multicéntricos, dado que es una enfermedad poco frecuente y es necesario el trabajo colaborativo de varios centros para conseguir el poder suficiente para identificar diferencias.

Si bien es un estudio retrospectivo, lo cual puede significar pérdida de datos e imposibilidad de evaluar variables confusoras, los datos utilizados para el estudio se extrajeron del Registro Institucional de Amiloidosis, en el cual se recolectan de manera sistemática y estandarizada las variables. Asimismo, esta institución cuenta con historial clínico electrónico, al cual tiene acceso autorizado el equipo de investigación para buscar datos faltantes o inconsistencias y datos administrativos que permitan el seguimiento del paciente. Asimismo, se realizó seguimiento mediante revisión semestral de historias clínicas.

La elevada prevalencia de amiloidosis cardíaca de este estudio podría explicarse en el siguiente contexto: la institución es un centro hospitalario de tercer nivel de complejidad, que recibe derivaciones de pacientes de todo el país e incluso de naciones vecinas como Bolivia, con elevada frecuencia de mutaciones génicas causantes de ATTRv; esta cohorte incluyó a pacientes con amiloidosis AA, más común en los países en vías de desarrollo. Si bien la afectación principal de estos pacientes es renal, un 7\% mostró compromiso cardíaco; el diagnóstico de amiloidosis cardíaca avanzó en los últimos años debido a la introducción de métodos de imágenes como el ecocardiograma con contracción longitudinal global (STRAIN), la resonancia cardíaca y el centellograma con pirofosfato, los cuales tienen patrones propios que permiten establecer el diagnóstico de amiloidosis cardíaca; por último, en este centro se realiza la identificación de compromiso cardíaco mediante métodos de imágenes a todo paciente con diagnóstico de amiloidosis.

La sobrevida global de los pacientes con amiloidosis cardíaca de esta cohorte es similar a la de otros estudios. Esto debe interpretarse en el siguiente contexto: el porcentaje de pacientes con amiloidosis $A L$ es del $51 \%$, mientras que en el estudio de Arnt, et al. es del $73 \%$. El subtipo de amiloidosis AL posee peor pronóstico cuando hay compromiso cardíaco, en parte por la toxicidad de las cadenas ligeras sobre los miocitos. El estudio de Czobor, et al., evaluó a 198 pacientes con compromiso cardíaco, de los cuales 183 tenían insuficiencia cardíaca al momento del diagnóstico. En esta institución se estudia el compromiso cardíaco mediante métodos diagnósticos de imágenes que permiten anticipar el diagnóstico de enfermedad cardíaca antes de que el paciente presente signos clínicos o gran compromiso. La amiloidosis sistémica en general y el compromiso cardíaco en particular se subdiagnostican a menudo o de forma tardía en fases avanzadas, lo que impide instituir tratamientos adecuados por la toxicidad de los fármacos y el deterioro orgánico de los pacientes.

En cuanto a los factores relacionados con mortalidad, faltó poder para hallar diferencias entre los distintos grupos de amiloidosis e incluso para el compromiso cardíaco; sólo se encontraron diferencias en el HR ajustado por edad. Esto tiene sentido en el contexto de 
un paciente de mayor edad con más probabilidades de morir por carga de enfermedades, al margen de su afectación. Asimismo, una cuarta parte de los pacientes de esta cohorte posee amiloidosis ATTRwt, tiene $100 \%$ de compromiso cardíaco y corresponde al grupo más añoso, lo cual puede explicar en parte estos resultados.

Por último, el análisis de regresión de riesgos competitivos arrojó un sub-HR con intervalo de confianza que incluye la no diferencia. Sin embargo, esto debe interpretarse sin perder de vista el bajo número de eventos competitivos, con criterios de elegibilidad para trasplante poco estrictos que incluyen a pacientes con compromiso sistémico grave y por tanto peor pronóstico global.

\section{Conclusión}

Esta investigación es un trabajo inicial que sienta las bases para futuros protocolos y muestra cómo el compromiso cardíaco es un factor pronóstico importante para la mortalidad en pacientes con amiloidosis. Es esencial conducir más estudios para determinar el diagnóstico temprano que permita instituir tratamientos oportunos.

\section{Financiamiento}

Los autores no recibieron patrocinio alguno para llevar a cabo este artículo.

\section{Conflicto de intereses}

Los autores declaran no tener ningún conflicto de intereses.

\section{Responsabilidades éticas}

Protección de personas y animales. Los autores declaran que para esta investigación no se han realizado experimentos en seres humanos ni en animales.

Confidencialidad de los datos. Los autores declaran que han seguido los protocolos de su centro de trabajo sobre la publicación de datos de pacientes.

Derecho a la privacidad y consentimiento informado. Los autores declaran que en este artículo no aparecen datos de pacientes.

\section{Bibliografía}

1. Banypersad SM, Moon JC, Whelan C, Hawkins PN, Wechalekar AD. Updates in cardiac amyloidosis: a review. J Am Heart Assoc. 2012;1:e000364.

2. Karafiatova L, Pika T. Amyloid cardiomyopathy. Biomed Pap Med Fac Univ Palacky Olomouc Czech Repub. 2017;161:117-127.

3. Di Giovanni B, Gustafson D, Delgado DH. Amyloid transthyretin cardiac amyloidosis: diagnosis and management. Expert Rev Cardiovasc Ther. 2019:17:673-681.

4. Blancas-Mejía LM, Ramírez-Alvarado M. Systemic amyloidoses. Annu Rev Biochem. 2013;82:745-774.

5. González-López E, López-Sainz Á, García-Pavía P. Diagnóstico y tratamiento de la amiloidosis cardíaca por transtiretina. Progreso y esperanza. Revista Española de Cardiología. 2017:991-1004. doi:10.1016/j.recesp.2017.05.018

6. Benson MD, Buxbaum JN, Eisenberg DS, Merlini G, Saraiva MJM, Sekijima $Y$, et al. Amyloid nomenclature 2018: recommendations by the International Society of Amyloidosis (ISA) nomenclature committee. Amyloid. 2018:215-219. doi:10.1080/13506129.2018.1549825

7. Mohty D, Damy T, Cosnay P, Echahidi N, Casset-Senon D, Virot P, et al. Cardiac amyloidosis: updates in diagnosis and management. Arch Cardiovasc Dis. 2013;106: 528-540.

8. Kyle RA, Larson DR, Kurtin PJ, Kumar S, Cerhan JR, Therneau TM, et al. Incidence of AL amyloidosis in Olmsted County, Minnesota, 1990 through 2015. Mayo Clinic Proceedings. 2019. doi:10.1016/j.mayocp.2018.08.041

9. Merlini G, Stone MJ. Dangerous small B-cell clones. Blood 2006:108:2520-2530.

10. Falk RH. Diagnosis and management of the cardiac amyloidoses. Circulation. 2005:2047-2060. doi:10.1161/circulationaha.104.489187

11. Rapezzi C, Lorenzini M, Longhi S, Milandri A, Gagliardi C, Bartolomei I, et al. Cardiac amyloidosis: the great pretender. Heart Fail Rev. 2015;20:117-124.

12. Amiloidosis cardíaca-Artículos-IntraMed. [cited 29 Jun 2020]. Available: https://www.intramed.net/contenidover.asp?contenidoid=75702

13. Said SM, Sethi S, Valeri AM, Leung N, Cornell LD, Fidler ME, et al. Renal amyloidosis: origin and clinicopathologic correlations of 474 recent cases. Clin J Am Soc Nephrol. 2013;8:1515-1523.

14. Aguirre MA, Boietti BR, Nucifora E, Sorroche PB, de Quirós FGB, Giunta $\mathrm{DH}$, et al. Incidence rate of amyloidosis in patients from a medical care program in Buenos Aires, Argentina: a prospective cohort. Amyloid. 2016:184-187. doi:10.1080/13506129.2016.1207626

15. Dubrey SW, Cha K, Anderson J, Chamarthi B, Reisinger J, Skinner M, et al. The clinical features of immunoglobulin light-chain $(A L)$ amyloidosis with heart involvement. QJM. 1998:91:141-157.

16. $\mathrm{Ng} \mathrm{B}$, Connors LH, Davidoff R, Skinner M, Falk RH. Senile systemic amyloidosis presenting with heart failure: a comparison with light chain-associated amyloidosis. Arch Intern Med. 2005;165:1425-1429.

17. Oerlemans MIFJ, Rutten KHG, Minnema MC, Raymakers RAP, Asselbergs $\mathrm{FW}$, de Jonge N. Cardiac amyloidosis: the need for early diagnosis. Neth Heart J. 2019;27:525-536.

18. Cornwell GG $3^{\text {rd }}$, Murdoch WL, Kyle RA, Westermark P, Pitkänen $P$ Frequency and distribution of senile cardiovascular amyloid. A clinicopathologic correlation. Am J Med. 1983;75:618-623.

19. Westermark $P$, Sletten $K$, Johansson $B$, Cornwell GG $3^{\text {rd }}$. Fibril in senile systemic amyloidosis is derived from normal transthyretin. Proc Natl Acad Sci USA. 1990;87:2843-2845

20. Shah KB, Inoue $Y$, Mehra MR. Amyloidosis and the heart: a comprehensive review. Arch Intern Med. 2006:166:1805-1813.

21. Gertz MA, Comenzo R, Falk RH, Fermand JP, Hazenberg BP, Hawkins $\mathrm{PN}$, et al. Definition of organ involvement and treatment response in immunoglobulin light chain amyloidosis $(A L)$ : a consensus opinion from the $10^{\text {th }}$ International Symposium on Amyloid and Amyloidosis, Tours, France, 18-22 April 2004. Am J Hematol. 2005;79: 319-328.

22. Lee M-H, Lee S-P, Kim Y-J, Sohn D-W. Incidence, diagnosis and prognosis of cardiac amyloidosis. Korean Circulation Journal. 2013:752. doi:10.4070/kcj.2013.43.11.752

23. Aguirre MA, González Bernaldo de Quirós F. Afección cardíaca en pacientes con amiloidosis. Revista del Hospital Italiano de Buenos Aires. 2014;34:36-40.

24. Czobor P, Hung Y-Y, Baer D, McGlothlin D, Weisshaar D, Zaroff J. Amyloid cardiomyopathy in a large integrated health care system. American Heart Journal. 2019:42-52. doi:10.1016/j.ahj.2019.06.008

25. Kristen AV, Perz JB, Schonland SO, Hegenbart U, Schnabel PA, Kristen $\mathrm{JH}$, et al. Non-invasive predictors of survival in cardiac amyloidosis. Eur J Heart Fail. 2007:9:617-624. 